\title{
Avalanches in the growth of stress-induced martensites
}

\author{
Lluís Carrillo and Jordi Ortín \\ Departament d'Estructura i Constituents de la Materia, Facultat de Física, Universitat de Barcelona, \\ Diagonal 647, E-08028 Barcelona, Catalonia, Spain
}

(Received 12 May 1997)

\begin{abstract}
We present an experimental study of phase-transition avalanches during the stress-induced formation of martensite in a $\mathrm{Cu}-\mathrm{Zn}-\mathrm{Al}$ alloy, with particular attention to the effect of cycling. We have analyzed statistically the amplitudes, durations, and energies of the thermal events accompanying the transition, and found that these magnitudes distribute according to power laws in the first fifty cycles, within experimental error, with exponents $\alpha=2.3 \pm 0.2, \tau=2.9 \pm 0.7$, and $\epsilon=1.8 \pm 0.3$, respectively. However, the extent of power-law behavior is reduced by at least one decade after five hundred transition cycles, and the system is seen to become progressively subcritical. We present a method of measuring the distribution of energy barriers encountered along the transition, which in our case is found to spread over more than four orders of magnitude. Finally, we have observed that repeated cycling leads to a statistical reproducibility of transformation trajectories, and to the remarkable correlation between the cycle-to-cycle trajectory fluctuations and the cycle-averaged response function of the system observed recently in magnetic systems [J.S. Urbach, R.C. Madison, and J.T. Markert, Phys. Rev. Lett. 75, 4694 (1995)]. [S0163-1829(97)09041-3]
\end{abstract}

\section{INTRODUCTION}

The dynamics of athermal first-order phase transitions, and the related phenomena of hysteresis and avalanches, have been subjects of increasing interest in the past years. ${ }^{1}$ Typically, the systems considered here display a complex free energy landscape in configuration space, with a multiplicity of metastable states. The energy barriers between configurations are so large that these systems are practically insensitive to thermal fluctuations (hence the name athermal). Consequently, the phase transition must be driven by an external field, which modifies the relative height of energy minima until an energy barrier is effectively suppressed and the system can explore a new configuration. Often this new configuration, on its turn, is unstable with respect to other neighboring configurations that were not previously accessible, and the result is an evolution by avalanches between metastable states. Macroscopically, the avalanches give rise to sudden jumps of the order parameter under smooth evolutions of the driving field. The resulting phenomenology is very interesting: the transformation trajectories (plots of order parameter against driving field) are insensitive to the rate of scanning the field, but they strongly depend on previous history and in many instances give rise to partial hysteresis cycles displaying memory of previous return points.

These transitions are rather common. Examples include typical first-order phase transitions, such as ferromagnetic transitions driven by an external magnetic field, ${ }^{2}$ and diffusionless structural phase transitions (martensitic transformations) driven either by temperature or by an external stress field. ${ }^{3}$ The same behavior has also been observed in a variety of other situations, such as liquid and gas adsorption by porous solids, ${ }^{4,5}$ the motion of charge-density waves, ${ }^{6}$ spin transitions, ${ }^{7}$ and cellular automata models of extended dissipative systems. ${ }^{8}$

In this work we focus on the dynamics of the athermal martensitic transformation (MT) of a single crystal alloy, driven by an external uniaxial stress. A martensitic transformation (MT) is a diffusionless (purely displacive), structural first-order phase transition exhibited by a large family of metallic alloys. This kind of transition has been thoroughly studied in the past two decades by physicists and metallurgists, particularly regarding questions such as the structural instability leading to nucleation of martensite, the relative stability between the high temperature phase and different martensitic structures, and the unusual thermomechanical properties (pseudoelasticity and shape-memory) that arise from the MT in some of the alloys mentioned.

In the MT, the parent phase has a higher symmetry than the product phase. Therefore, a single crystal parent phase gives rise to a multiplicity of differently oriented, symmetry related variants of the product phase. The shape and volume differences between the unit cells of the two lattice structures, along the diffusionless transformation, produces coherency stresses on the products as they form, which results in a selection process for the variants of the product phase: the variants formed are those which minimize the overall strain energy. In noble metal based alloys, as the one studied in this work, the lattice distortion is not too large (transformation strain around $10^{-2}$ ) and the stresses can be relieved by elastic deformation of the two phases; the result is a topologically and elastically reversible (thermoelastic) transition. ${ }^{9}$ Nevertheless, the presence of coherency stresses due to the transformation strain, together with stresses originating from quenched defects (vacancies, dislocations, impurities), gives rise to long-lived polyvariant and parent-product arrangements of domains, separated from other possible arrangements by large energy barriers. Hence the system must be continuously driven externally to resume the MT at each transformation stage and carry it progressively to completion.

The presence of some kind of structural disorder, such as coherency stresses in the case of MT, is a generic feature of systems with a rugged free energy landscape and athermal 
behavior. Structural disorder can be added to spin models of a first-order phase transition in the form of random fields, like in the random field Ising model (RFIM), ${ }^{1}$ random-field Ising model with vacancies, (RFIMV), ${ }^{10}$ and random field Blume-Emmery-Griffiths model (RFBEG), ${ }^{11}$ or in the form of random bonds like in the random bond Ising model (RBIM). ${ }^{12}$ To study athermal transitions, these models have been investigated by driving them with an external magnetic field at $T=0$ (thermal fluctuations neglected). By changing the concentration of quenched disorder, it has been found that all the models exhibit a disorder-induced phase transition at which amplitudes and durations of avalanches distribute according to power laws. In addition, several scaling relations have been found to hold in the critical region, ${ }^{1,10}$ and it has been possible to associate universality classes to this kind of models. ${ }^{13}$

Extensive experimental work has also been carried out recently. In magnetic materials the research has centered on the statistical properties of Barkhausen noise, a direct measure of the magnetization jumps at avalanches. ${ }^{14}$ In MT, correspondingly, experiments have focused on the statistical properties of acoustic emission events (AE) associated with the release of elastic waves at avalanches. ${ }^{15}$ Other experimental studies have dealt with superconducting flux lines ${ }^{16}$ and $\mathrm{He}$ adsorption in Nuclepore. ${ }^{5}$

In spite of the ample research carried out, a number of open questions remain. An important one is the reason why the systems are found close to the critical concentration of disorder (as shown by the fact that avalanches distribute according to power laws), without having carefully tuned the disorder concentration. This question has been addressed here for the particular case of a stress-induced MT. We study how the statistical distribution of transformation events is modified, upon cycling, by an increasing number of defects acting as pinning centers of the martensite-austenite interfaces. In particular, whether the system naturally settles on a critical state characterized by the absence of length and time scales, as observed by Vives et al. ${ }^{15}$ for the thermallyinduced MT of a $\mathrm{Cu}-\mathrm{Zn}-\mathrm{Al}$ alloy brought already to a reproducible transformation trajectory by repetitive cycling, or it tends to leave such particular state. To this purpose, we have prepared a system in a well defined state, by means of a particular thermal treatment, and progressively increased the amount of disorder in the system by subjecting it to a large number of transformation cycles. Cycling through the MT is known to increase the concentration of crystalline defects (mainly dislocations) during a number of cycles, up to a saturation value. Actually, the spontaneous generation of a large number of dislocations with a particularly defined orientation during thermomechanical cycling (a procedure usually called training) is acknowledged to be the physical origin of the shape-memory effect in the kind of materials that we are considering. Then, using thermal techniques, we have studied the evolution of both the transformation trajectories and the statistical distributions of avalanches with cycling.

The paper is organized in the following way: Section II provides a general introduction to the stress-induced MT, with emphasis on the alloy used in the present research and the requirements imposed on the tensile machine. Section III presents the experimental setup (loading machine, electronics, sample used, and thermal treatment), and Sec. IV the experimental procedures (calibration of the machine and data analysis). Our results are presented and discussed in Sec. V. We study the evolution of transformation trajectories with cycling, the corresponding modification in the distributions of amplitudes, durations and energies of the recorded signals, and their power spectrum. Next, we analyze the distribution of energy barriers along the transition. Finally, we explore a possible relationship between fluctuations of the order parameter (with repeated cycling) and dissipation in the system. Section VI gives the conclusions of our work.

\section{THE STRESS-INDUCED MT}

Many copper-based alloys with electronic concentrations per atom close to the eutectoid concentration $(e / a=1.48)$, including the $\mathrm{Cu}-\mathrm{Zn}-\mathrm{Al}$ crystal used in the present work, present a stable body-centered cubic structure at high temperatures, called $\beta$ or parent phase. This structure can be retained as a metastable phase at moderate temperatures by means of adequate thermal treatments. Depending on composition, temperature and external stress, the parent phase experiences a first-order diffusionless phase transition (MT) to a close-packed structure called $M$ or martensite. The change in crystal structure is essentially accomplished by a homogeneous shear on the $\{110\}$ planes of the parent phase, along the $\langle 1 \overline{1} 0\rangle$ direction. Generally speaking, the $M$ phase is more stable than the $\beta$ phase at lower temperatures and higher tensile stresses.

One particular case of interest is the parent-to-martensite transformation of a single crystal, induced by application of an uniaxial stress. ${ }^{18}$ Initially, the bcc crystal responds elastically to the applied stress; at a critical stress level the martensitic transformation takes place and, since the new structure has a different shape, the crystal is able to accommodate large strains at nearly constant stress. When all the crystal has transformed into the close-packed phase, this phase responds again elastically to the applied stress. If the applied stress is then reduced, below a new critical stress level the martensite phase reverses to the parent phase, and the large strains achieved in the phase transition are fully recovered. The overall stress-strain behavior (usually called pseudoelastic) is highly nonlinear and hysteretic. Examples can be found in Ref. 3.

It is important to note here that most experiments on pseudoelasticity have been carried out on screw-driven tensile machines which control strain instead of stress, or in servohydraulic machines which control stress by a feedback adjustment of strain. This is not appropriate when dealing with the dynamics of the transformation in single crystals, because growth velocities at a parent/martensite interface are in the range 500 to $3500 \mathrm{~m} / \mathrm{s}$, comparable to the speed of sound in the material, ${ }^{19}$ and corresponding crystal strains can be as large as 0.1 to $1 \%$. Since machines of the kind mentioned cannot accommodate the transformation strain nearly instantaneously, they prevent spontaneous motion of the interfaces and give rise to abrupt fluctuations in stress that are not consubstantial to the phase transition. The right way to ensure that the stress field in the crystal follows the applied external stress is to use an infinitely soft machine that applies a dead load to the specimen and allows for free accommodation of the new shape. In addition, it is important to recall that the 


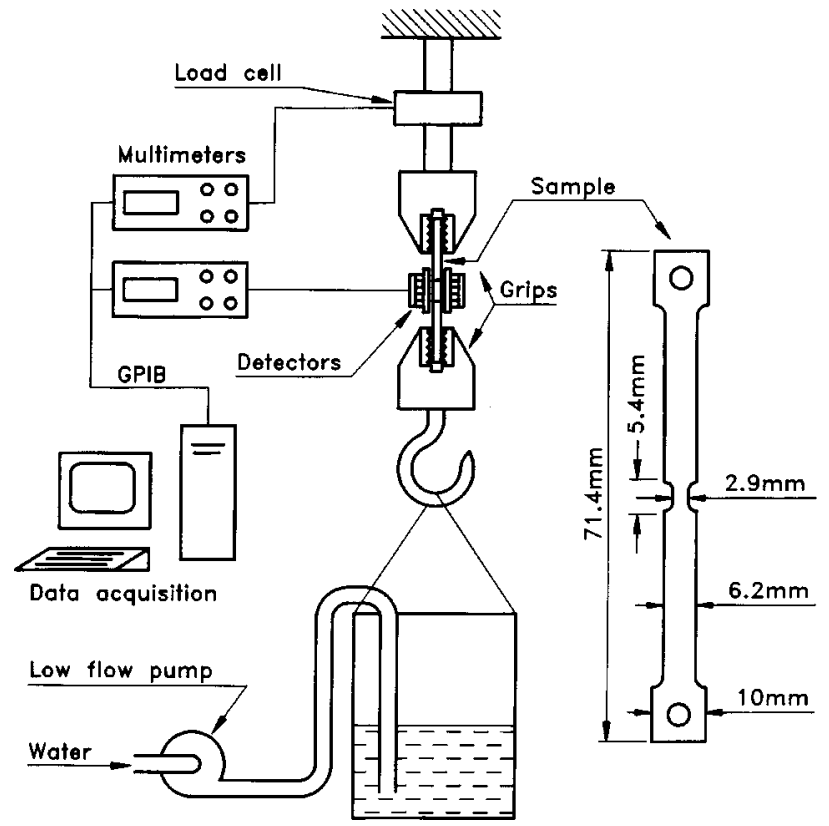

FIG. 1. Schematic view of the experimental setup, and specimen dimensions.

structural change is essentially a homogeneous shear that, unavoidably, produces a lateral displacement of the tensile axis. The machine must be designed also to allow for this displacement. Both requirements have been taken into account to build the loading machine used in the present investigation, described in the next section.

\section{EXPERIMENTAL SETUP}

Figure 1 shows a scheme of the machine employed. The tensile sample is attached to the machine by means of two grips. The upper grip is screwed to a load cell hanging from the laboratory ceiling; the lower grip carries a water tank that plays the role of an applied dead load. The load can be increased or decreased by supplying or removing water from the tank, using a low flow pump. Typical flow rates are low, about $2.5 \mathrm{~cm}^{3} / \mathrm{s}$, to make sure that the crystal is carried quasistatically through the transition (i.e., the applied stress does not change appreciably in a transformation event), and to allow for dissipation of the transformation latent heat to avoid changes in the temperature of the sample. A complete run starts always from a load of $0.00 \mathrm{~N}$, in the bcc phase, and goes up to a maximum load of $150.00 \mathrm{~N}$, at which the whole tensile part of the sample is transformed to martensite. Measurements are recorded in an overall interval of about $10 \mathrm{~N}$ beginning at the detectable onset of the phase transition.

The parameters measured are the applied load (external driving) and the thermal power released in the MT (system response). The applied load is monitored by a load cell attached to the upper end of the specimen, which provides a linear signal of $0.045 \mathrm{mV} / \mathrm{N}$ in the range from 0 to $6670 \mathrm{~N}$. This signal is read every $20 \mathrm{~s}$ by a multimeter HP 3441A, working at a resolution of $\pm 0.1 \mu \mathrm{V}$. Thermal power pulses from the specimen are detected by two Peltier thermobatteries, each one comprising 66 junctions in a useful area of $11.4 \times 9.0 \mathrm{~mm}^{2}$, connected in series. A Peltier thermobattery transforms a temperature gradient perpendicular to the bat- tery into a proportional dc voltage. In our setup, the tensile portion of the sample is sandwiched between the two thermobatteries, and the outer faces of the thermobatteries are thermally connected to a copper block. A thermally conducting paste is used to improve the thermal contact. The ensemble (grips, transducers and sample) is wrapped in plastic to minimize external thermal disturbances. A second multimeter HP 3441A, working now at a resolution of $\pm 1 \mu \mathrm{V}$, reads the dc signal from the thermobatteries at a sampling rate of $21.066 \pm 0.001 \mathrm{~Hz}$. The readings of both multimeters are sent through an IEEE-488 interface bus to a PC, where they are stored for future analysis.

The sample used is a single crystal of nominal composition $\mathrm{Cu}-19.4 \mathrm{Zn}-13.1 \mathrm{Al}($ at. \%), corresponding to an electronic concentration $e / a=1.46$. The crystal has been sparkmachined to the form of a flat tensile specimen, of thickness $0.81 \mathrm{~mm}$, whose shape and dimensions are shown in Fig. 1. The stress-induced phase transition is restricted to the region of smallest cross section (in the center of the specimen), since applied stresses are much higher there than in the rest of the specimen.

To start cycling from a well defined austenitic state, the sample has been subjected to an initial thermal treatment consisting of annealing in the $\beta$ phase for $20 \mathrm{~min}$ at $850{ }^{\circ} \mathrm{C}$ followed by quenching in water at room temperature. This thermal treatment is suitable to remove any memory of the previous thermomechanical history of the sample and to retain the $\beta$ phase as a metastable phase at room temperature (in the absence of applied load) with a relatively low concentration of quenched-in defects.

The MT is induced by application of an uniaxial load at room temperature. Martensite is observed to nucleate in the form of very thin plates, typically at one edge of the specimen, and quickly grow up to the whole width of the crystal (Fig. 2). The growth direction of the martensitic variant selected forms an angle of $58.5^{\circ}$ with the direction of the applied load. After cycling through the MT for more than 500 times, the crystal does not show any sign of structural damage at the scale of the optical microscope.

\section{EXPERIMENTAL PROCEDURE}

To perform a static calibration of the apparatus, the actual sample has been replaced by a pure copper sample of similar dimensions, which carries inside its tensile part a heating resistor $(69 \Omega)$ made of silk-insulated constantan wire. A constant thermal power dissipated in the copper sample gives rise to a steady heat flow from sample to transducers, once all transients disappear. On its turn, the steady heat flow results in a constant voltage output of the thermobatteries. The static calibration consists on determining the relation between the thermal power released and the corresponding constant voltage output in the steady state. Our results show that in the range of thermal powers explored, between 0.03 $\mathrm{mW}$ and $0.88 \mathrm{~mW}$, the dependence is linear and given by $P=3.5747 V+0.01$, where $P$ is the thermal power in $\mathrm{mW}$ and $V$ is the voltage output in $\mathrm{mV}$.

A first order approach to the dynamic response of the experimental apparatus is given by the main time constant of the transducers response to an impulsional thermal power dissipation. This time constant can be determined by a least- 


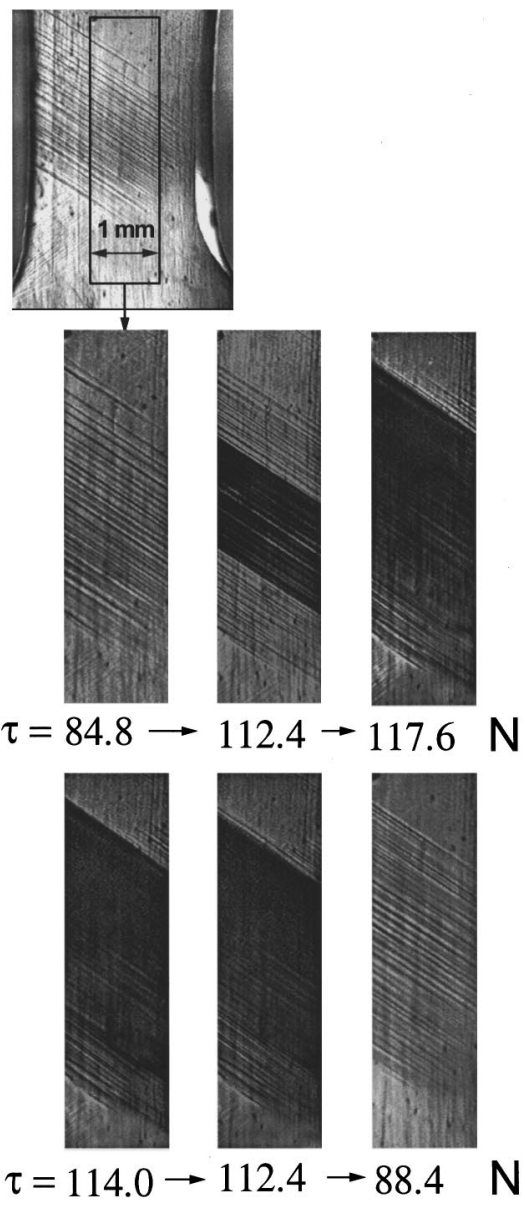

FIG. 2. Evolution of the two-phase microstructure as a function of applied load $\tau$, during a loading-unloading cycle. Martensite plates appear dark.

squares fit to the exponential decay of an isolated voltage pulse (presumably resulting from a nearly impulsional thermal power dissipation). An average over a number of trials on pulses of different sizes produces $\tau_{1}=5.2 \pm 0.1 \mathrm{~s}$.

Before attempting a statistical analysis of the experimental recordings, raw data (voltage output as a function of time) has been digitally filtered from the main time constant of the measuring device to compensate for its thermal inertia, and corrected for baseline modulations caused by low frequency variations of the laboratory temperature. The result of this numerical treatment is shown in Fig. 3.

A threshold of $0.19 \mathrm{mV}$ has been used to discriminate valid signals from background noise. The duration of an event has been measured as the time elapsed between the last minimum found before a signal crosses threshold on rising and the first minimum found once this same signal falls again below threshold. The maximum of the signal recorded in the interval defined by the two crossings is taken as the amplitude of the event. A silent period in the train of signals is defined as the time elapsed between two consecutive events, defined by the second crossing of the first event and the first crossing of the second event. Finally, to analyze the influence of electric noise on the power spectrum of the signals, we consider as noise periods the time intervals in which the signal never rises above threshold and the time average

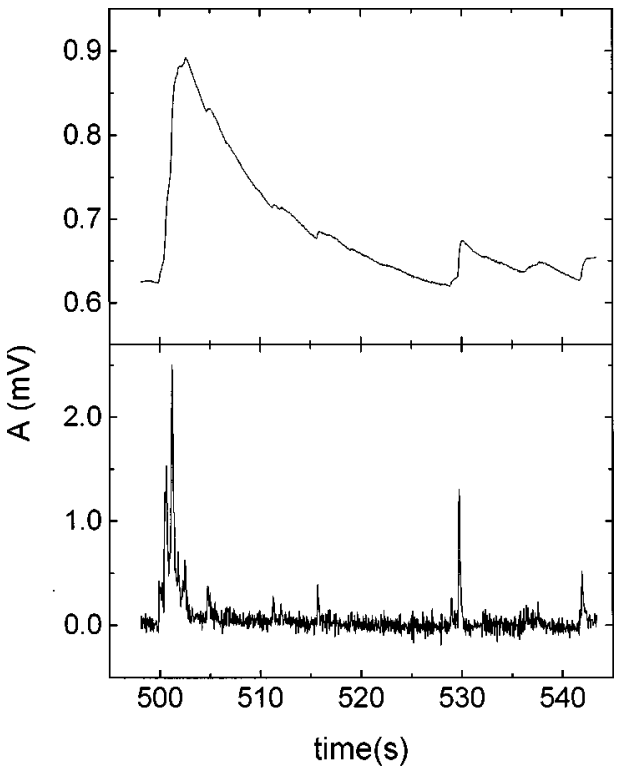

FIG. 3. Piece of recorded signal (top), and the same signal after having filtered the main time constant of the experimental device (bottom).

of the voltage signal remains below $0.002 \mathrm{mV}$ in absolute value.

Where necessary, the filtered voltage signal $V$ (in $\mathrm{mV}$ ) has been converted into a thermal power signal $W$ (in $\mathrm{mW}$ ) using the static calibration of the apparatus, and the time scale $t$ (in s) has been translated into a load scale $\tau$ (in $N$ ) by proper interpolation of the load data recorded as a function of time.

\section{RESULTS AND DISCUSSION}

Considering that dissipative effects are about two orders of magnitude smaller than the latent heat of transformation, ${ }^{20}$ it is a good approximation to conside that the thermal energy released by the sample during the MT, $E$, is proportional to the volume fraction of material transformed, $x$. Thus, thermal pulses of large area in the experimental train correspond to transformations of a large fraction of the sample and, presumably, to extensive displacements of the austenitemartensite interphases. In this scenario, the amount of transformation $x$ at a given applied load $\tau$ is proportional to the overall energy released by the sample up to that load, obtained by integration of the temporal train of thermal power pulses, i.e., $x \propto E=\int_{0}^{t} W(t) d t$. The resulting curve, overall energy released $E$ (extensive variable) vs load $\tau$ (intensive variable), represents the isothermal trajectory of the specimen through the stress-induced MT, at room temperature.

Figure 4 shows the evolution of this trajectory with cycling. Between cycles 3 and 40 the pattern is very similar: the MT starts by small transformation events, at small loads, which give rise to an ascending staircase trajectory; then, at a threshold value of the applied load, a large amount of energy is released in one or very few events, and almost all the tensile part of the specimen transforms to the new phase; from here on, the remaining transformation is completed gradually, by relatively small events. Upon cycling through the MT, however, very large transformation events tend to 


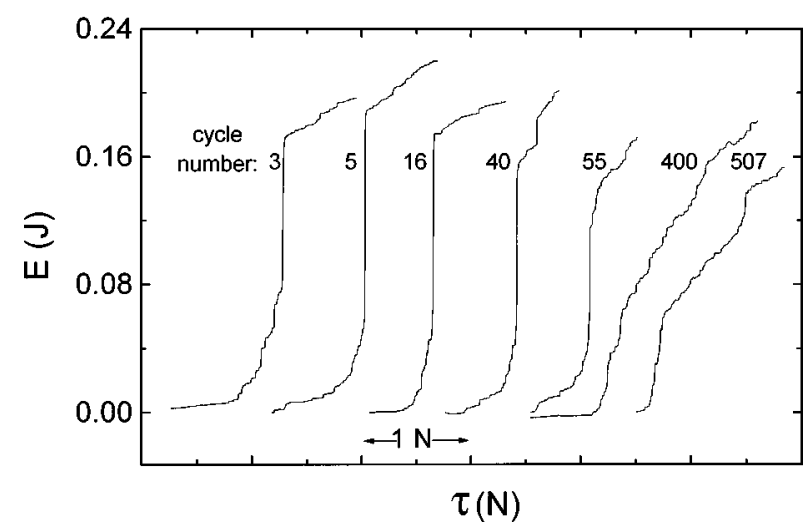

FIG. 4. Transformation trajectories obtained by integration of the filtered thermal signal, upon loading, for increasing number of cycles. The curves have been shifted arbitrarily along the horizontal axis to avoid overlapping.

break up into smaller ones. From cycle 400 onwards, the trajectory of transformation becomes a structured staircase at all loads in the transformation range, and becomes more and more reproducible with an increasing number of cycles.

The joint probability distribution of amplitudes and durations of the thermal events, $p(A, T)$, is shown in Fig. 5. In order to improve the statistics we have averaged together the results of nearly consecutive cycles, corresponding to two extreme cases: on top the sample has undergone a limited number of cycles, up to cycle number 40 (LIM), while on bottom it has been severely cycled for more than 500 cycles (SEV). This figure is very useful to estimate the limits imposed by a windowing effect ${ }^{21}$ on the separate (marginal) distributions of amplitudes and durations, $p(A)$ and $p(T)$, defined as

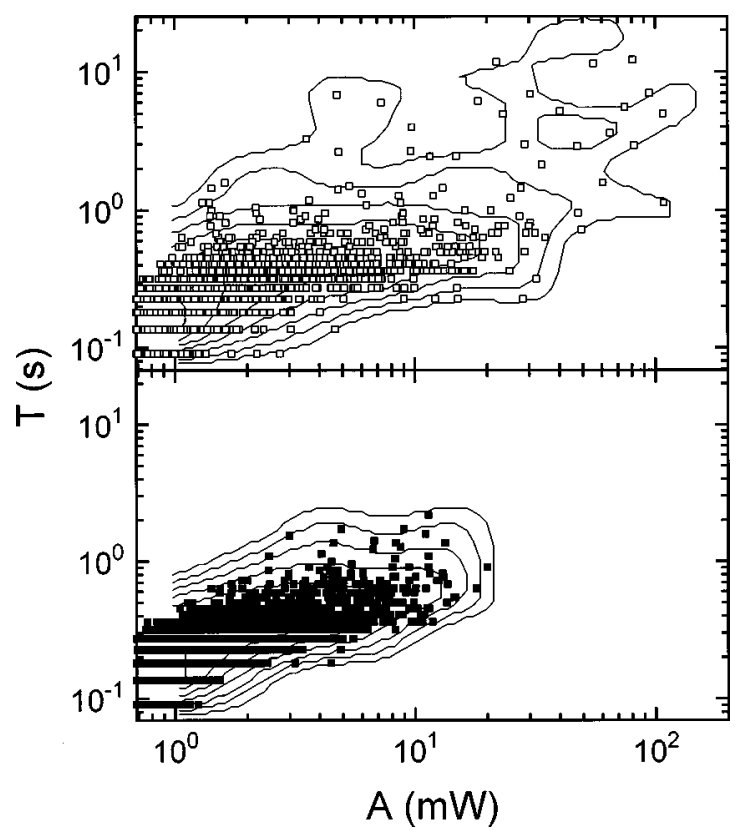

FIG. 5. Maps of the joint probability distribution of amplitudes and durations, in two extreme cases: LIM (top; cycle 2, 3, 4, 5, 6, 7, 8, 9, 10, 17, 23, 31, 41, and 56) and SEV (bottom; cycle 503, 504, $505,507,508,510,511,512,514$, and 515). The contour levels are logarithmically spaced, starting at $10^{\circ}$ (one event), and following with $10^{0.5}, 10^{1}, 10^{1.5}, 10^{2}, 10^{2.5}$, and $10^{3}$ events.

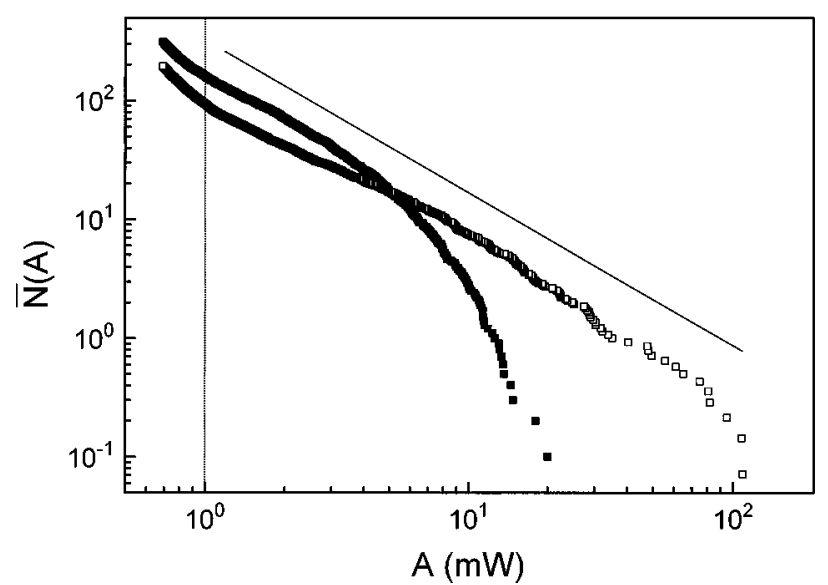

FIG. 6. Integrated distributions of amplitudes, averaged over the fourteen cycles of the LIM case (hollow squares) and over the ten cycles of the SEV case (solid squares). In the LIM case, the distribution can be reasonably fitted by a power law of the form $A^{-1.3}$ (solid line) for about two decades in amplitude. Windowing of the experimental data is relevant for amplitudes below the dotted line.

$$
\begin{aligned}
& p(A)=\int_{T_{\text {min }}}^{T_{\max }} p(A, T) d T, \\
& p(T)=\int_{A_{\text {min }}}^{A_{\max }} p(A, T) d A .
\end{aligned}
$$

We see from the figure that the statistics of amplitudes will be valid only for amplitudes larger than $1 \mathrm{~mW}$, since smaller amplitudes, although measurable in principle, correspond to events of durations too short to be resolved. Equivalently, the statistics of durations will be distorted for durations shorter than $0.4 \mathrm{~s}$ due to a lack of resolution in the corresponding amplitudes.

From $p(A)$ and $p(T)$, the integrated distributions $N(A)$ and $N(T)$ can be defined as

$$
\begin{aligned}
& N(A)=\int_{A}^{\infty} p(A) d A, \\
& N(T)=\int_{T}^{\infty} p(T) d T .
\end{aligned}
$$

$N(A)$ represents the number of events with an amplitude equal or higher than $A$, and $N(T)$ the number of events with a duration equal or longer than $T$. The interest of introducing these two distributions lies on the fact that they are not affected by the binning procedure used to represent them. Figures 6 and 7 present the experimental histograms of these distributions in the two extreme cases considered previously (LIM and SEV). The two figures show that the dominant effect of cycling is to modify the distributions for the largest amplitudes and durations, eliminating the corresponding events. The statistics of events of small and moderate amplitude and duration are not significantly altered. The joint histogram $p(A, T)$ (Fig. 5) confirms these two observations: rare, large events have disappeared after cycling, but most features of the joint distribution are not modified appreciably. 


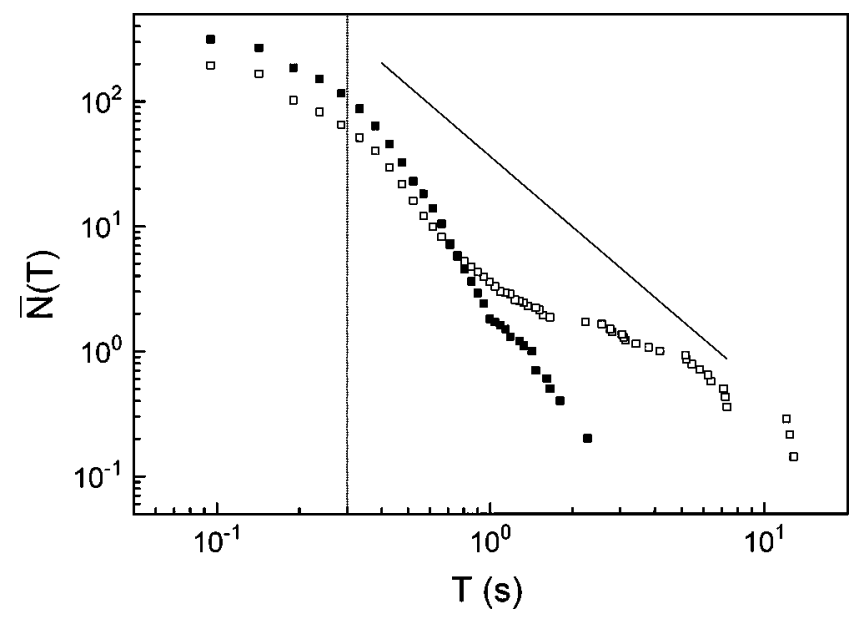

FIG. 7. Integrated distributions of durations, averaged over the fourteen transformation cycles considered in the LIM case (hollow squares) and over the ten cycles considered in the SEV case (solid squares). In the LIM case, above the limit of $0.4 \mathrm{~s}$ imposed by windowing of the experimental data (dotted line), a linear fit to the distribution has the form $T^{-1.9}$ (solid line).

The change of $N(A)$ (Fig. 6) is particularly remarkable. In the first cycles (LIM) the distribution follows a power law for two orders of magnitude of $A$. If we assume a dependence of the form $p(A) \sim A^{-\alpha}$, then $N(A) \sim A^{-(\alpha-1)}$ and from the linear fit shown in the figure we get $\alpha=2.3 \pm 0.2$. When the sample has been largely cycled (SEV) the powerlaw form of the distribution is cut off above half the first decade in $A$. This result points to the conclusion that the distribution of defects in the material sets up a limit to the size of martensite domains well below the limit imposed by the (finite) size of the system.

The behavior of $N(T)$ is similar (Fig. 7), though less clear. Our results show that events of durations between 3 and $12 \mathrm{~s}$ (the longest events detected) disappear with intensive cycling. If we assume again for the first cycles (LIM) a distribution $p(T) \sim T^{-\tau}$, then $N(T) \sim T^{-(\tau-1)}$ and from the linear fit shown in the figure we get $\tau=2.9 \pm 0.7$.

In the language of spatially extended dissipative systems, our results point to the conclusion that the dynamics of the system evolves with cycling from a critical state, characterized by a power law in the interval of amplitudes and durations not deformed by experimental windowing of the data $(1-100 \mathrm{~mW}$ and $0.4-10 \mathrm{~s})$, to a subcritical state in which the number of avalanches of large amplitudes (between 6 and $100 \mathrm{~mW}$ ) and durations (between 3 and $12 \mathrm{~s}$ ) is far below the number expected for a power law. In spin models with quenched disorder such as the RFIM or the RBIM (which are considered relevant here because they have been able to capture many other distinct features of athermal, externally driven first-order phase transitions ${ }^{1}$ ), the tendency of the distributions to become subcritical with an increasing number of cycles would be related to a widening of the distribution of random fields or bonds, induced by cycling. For a MT, the widening would translate into a relative increase of stronger pinning points or defects with cycling.

Our result that strong cycling takes the system out from the basin of attraction of criticality (where the different statistical distributions of transformation events are power laws or close to power laws), to a subcritical state, seems to be in contradiction with previous reports ${ }^{15}$ of power laws in the statistical distribution of acoustic emission events, recorded in the thermally-induced MT of a $\mathrm{Cu}-\mathrm{Zn}-\mathrm{Al}$ alloy which had been repeatedly cycled through the phase transition. The comparison is not straightforward, however, for a number of reasons: first, in the thermally-induced MT of a bcc single crystal martensite plates form in up to twenty-four possible different orientations in space, while in a stress-induced MT of the same crystal only one orientation is selected almost always. The different effective dimensionality of the two situations possibly has an influence on criticality. Second, the techniques used to characterize the transformation events in one case and in the other case not only look at different magnitudes (our thermal technique detects transition latent heat, proportional to transformed fraction, whereas acoustic emission detects the release of strain energy in the form of elastic waves at the transition) but their characteristic scales of observation differ in some orders of magnitude as well. Our thermal technique is able to detect all the large scale events, whereas acoustic emission is more sensitive to small scale events, at the price of a cutoff at large amplitudes due to the limited overall resolution of the digitizing oscilloscope. In this context, it is worth remembering that extensive computer simulations of the disorder-induced criticality of the RFIM (Ref. 1) have shown that the critical point displays such a large basin of attraction that, even thirty percent off it, the distributions of amplitudes and durations still display power laws over several decades.

Recently ${ }^{21}$ it was suggested that the joint probability distribution of amplitudes and durations in a problem of growth by avalanches could be described by a functional dependence of the form $p(A, T)=g\left(A / T^{x}\right) A^{\gamma} T^{\delta}$, from which a scaling relation $x(\alpha-1)=(\tau-1)$ follows. Inserting our values for $\alpha$ and $\tau$ leads to $x=1.3$, which means that the crest of the joint distribution $p(A, T)$ is close to a straight line in double logarithmic scale; this result is confirmed by inspection of Fig. 5 (top).

At the beginning of this section we mentioned that the energy contained in each thermal pulse (the area below the peak) is very approximately proportional to the transformed fraction in the event considered, i.e., $d x \propto W(t) d t$. The integrated distribution of event energies, averaged over a number of cycles, is shown in Fig. 8. Again, we consider the two extreme cases LIM and SEV. Beyond the lower limit imposed by the window effect, the distribution of LIM cycles is fairly well represented by a power law over three decades in energy. In this limit, the result shows unambiguously that the stress-induced MT gives rise to transformation events of all sizes: the transformed domains do not present a characteristic size scale. Conversely, when the sample has undergone a large number of cycles (SEV) there are no more events of the largest energies, and the third decade of the distribution disappears.

The dynamics of the system can be characterized as well by the statistics of energy barriers between consecutive metastable states. In a silent period between two transformation events the configuration of the system remains trapped in a metastable state, while the applied load modifies the energy landscape until an energy barrier disappears, triggering a new avalanche. Assuming that the applied load, $\tau$, couples linearly with the elongation of the crystal, it can be proved 


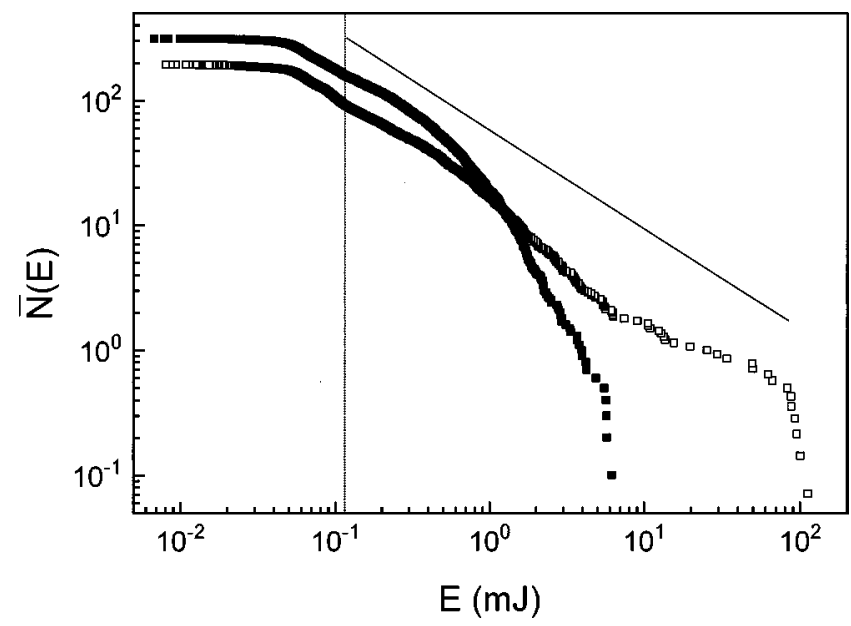

FIG. 8. Integrated distributions of event energies, averaged over the fourteen cycles of the LIM case (hollow squares) and over the ten cycles of the SEV case (solid squares). Above the dotted line, where windowing effects are negligible, the distribution in the LIM case can be approximately fitted to a power law of the form $E^{-0.8}$ over three decades in energy (solid line).

that $\Delta U$, the size of the energy barrier between two consecutive states, is proportional to $\left(\tau_{f}-\tau_{i}\right)$, the change in applied load between the corresponding transformation events. ${ }^{22}$ Hence, the statistics of the energy barriers encountered by the system along the MT can be derived from the load intervals measured in the silent periods. The integrated histogram of $\Delta U$ computed in this way is presented in Fig. 9 in semilogarithmic scale, for LIM and SEV cycles. The qualitative behavior of the two data sets is rather similar. It is interesting to notice that the number of large energy barriers is practically the same in the two limits studied, but that of medium and low energies has increased noticeably after five hundred cycles. ${ }^{23}$ This finding indicates that the distribution of barrier sizes does not keep a simple relation with the distributions of amplitudes and durations of transformation avalanches: high (low) energy barriers cannot be related directly to large (small) amplitude avalanches. The relative increase in the

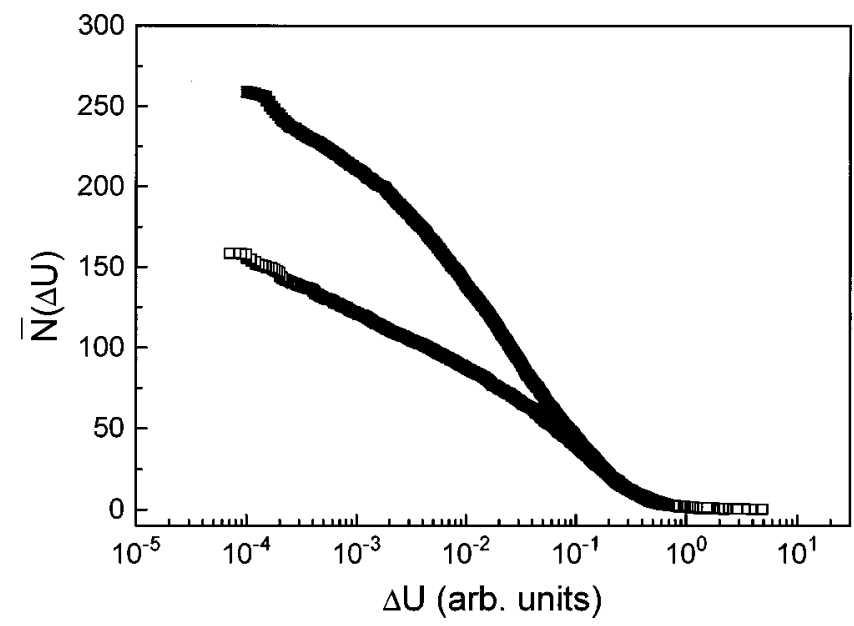

FIG. 9. Integrated distributions of energy barriers (measured by the intervals of applied load between transformation events), averaged over the LIM cycles (hollow squares) and the SEV cycles (solid squares).

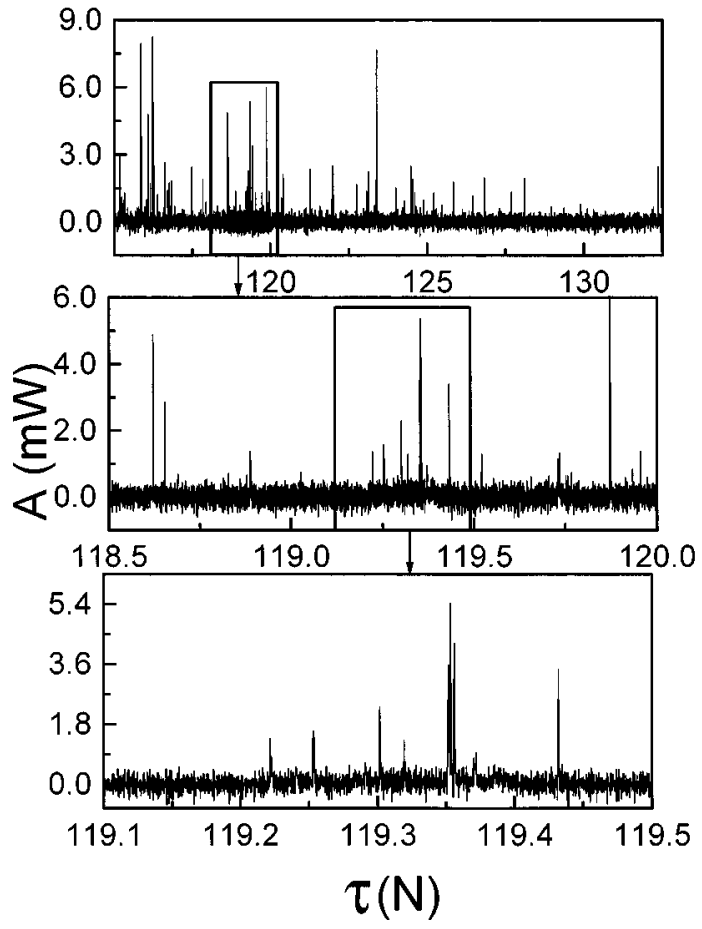

FIG. 10. Structure of the filtered thermal signal at different scales (cycle no. 503, loading).

number of low energy barriers, however, do seems to be associated with an increase in the number of strong pinning points and with the emergence of a subcritical behavior. These results suggest for a theoretical study of the correlation between energy barrier sizes and the statistical characterization of the disorder, in the framework of spin models with quenched disorder.

The observation of power laws in the statistical measures of the transformation events suggests a self-similarity of the thermal signals at different scales. An example of this rich structure is given in Fig. 10 and its integrated representation in Fig. 11. In this context, it is interesting to consider the power spectrum of the filtered signal, $c(\nu)$, computed as the discrete Fourier transform of the correlation function $c(t)$, defined by

$$
c(t)=\frac{1}{\Delta} \int_{0}^{\Delta} W(u) W(t+u) d u-\left(\frac{1}{\Delta} \int_{0}^{\Delta} W(u) d u\right)^{2},
$$

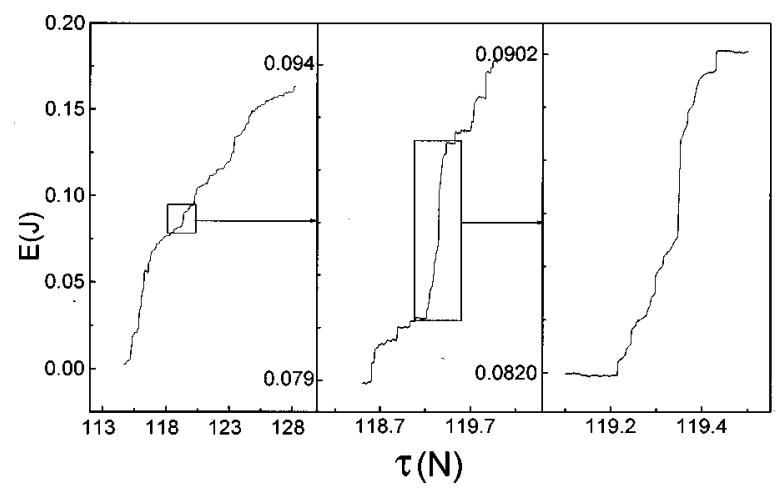

FIG. 11. Transformation trajectories observed at different scales, obtained from integration of the signals shown in the previous figure (cycle no. 503, loading). 


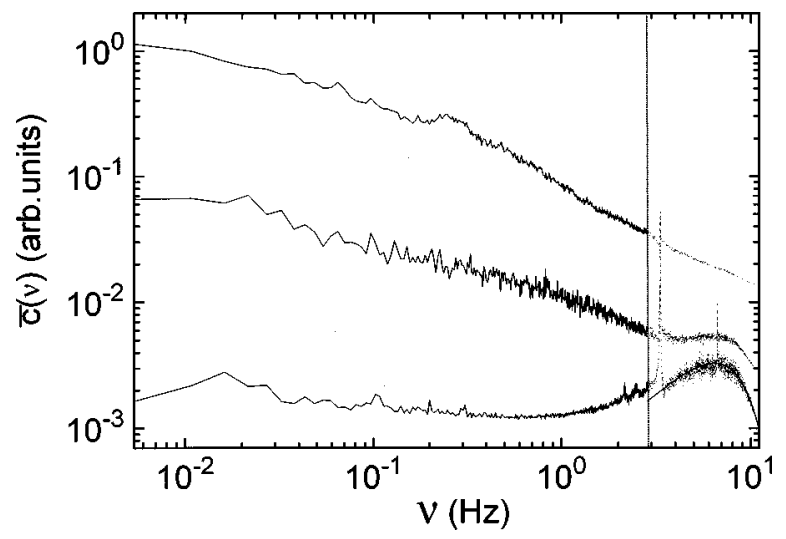

FIG. 12. Average power spectrum of the trains of thermal signals recorded upon loading. The top curve is the average for LIM cycles, and the middle one the average for SEV cycles. The bottom curve is the power spectrum of experimental noise, which above 3 $\mathrm{Hz}$ is well approximated by the power spectrum of gaussian noise (solid line). The vertical dotted line separates a region on the left, where the noise on the recorded signal can be considered white (solid curves), from a region on the right where the noise is Gaussian (dotted curves).

where it is assumed that $c(t) \simeq 0$ for $t>\Delta$. We have chosen $\Delta=180 \mathrm{~s}$ for sequences extending over $4000 \mathrm{~s}$. Figure 12 presents the power spectrum of the thermal signals for the two cases analyzed, LIM (top curve) and SEV (middle curve). In both cases we observe a $1 / f$ behavior over more than one decade in frequency. Part of the power spectrum in SEV cycles, however, is largely affected by electric noise on the recorded signal. This influence has been analyzed by computing the power spectrum of large noise periods (defined in the previous section). The result is given by the bottom curve in Fig. 12. The noise is white for frequencies below $1 \mathrm{~Hz}$ and Gaussian between 3 and $10 \mathrm{~Hz}$, and clearly the Gaussian noise is responsible for the deviations of the power spectrum from a $1 / f$ law at frequencies above $3 \mathrm{~Hz}$. Conversely, noise seems to have a negligible effect on the power spectrum in LIM cycles. The reason lies on the fact that $c(\nu)$ is drawn in logarithmic scale: the power spectrum for the first few cycles (LIM) is dominated by signals of large amplitude and duration, which liberate most of the energy, while after 500 cycles (SEV) the energy is released at much smaller scales, comparable to noise. In addition, we observe two sharp peaks at $3 \mathrm{~Hz}$ and $6.7 \mathrm{~Hz}$, attributed to uncontrolled external noise sources.

Interestingly, the range of frequencies explored in this experiment (centered about $1 \mathrm{~Hz}$ ) is four orders of magnitude below the range explored for a thermally-induced MT by ultrasonic techniques ${ }^{15}$ (centered about $10^{4} \mathrm{~Hz}$ ), and yet the power spectrum looks very similar. Two very different experimental techniques, therefore, looking at very different scale sizes, produce a unique picture of scale invariance over many decades.

Very recently, Urbach et al. ${ }^{17}$ discussed the possibility that an equivalent of the fluctuation-dissipation theorem would hold for extended out-of-equilibrium systems whose dynamics are dominated by static disorder. These authors studied the fluctuations of cycle-averaged Barkhausen jumps (magnetic avalanches) during nearly reproducible magnetic cycles in an Fe-Ni-Co ferromagnet. The fluctuations in mag-

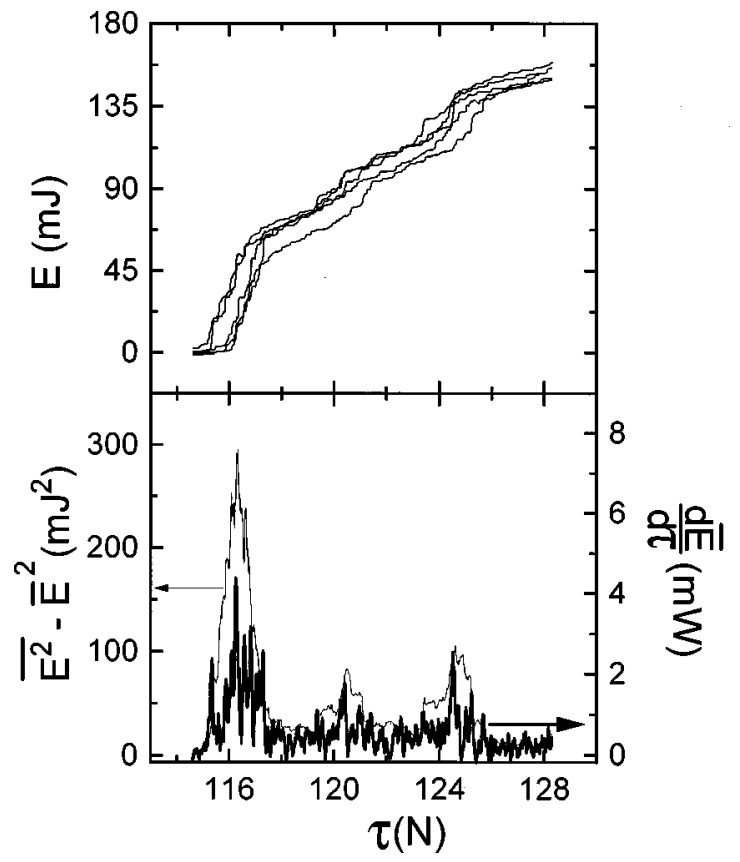

FIG. 13. Top: transformation trajectories for cycles 503, 507, 508, 513, and 514, upon loading. Bottom: correlation between the cycle-averaged fluctuations of the order parameter, $\overline{E^{2}}-\bar{E}^{2}$ (thin line), and the cycle-averaged response function, $d \bar{E} / d \tau$ (thick line), for the five trajectories considered above.

netization correlated strongly with the cycle-averaged magnetic susceptibility, particularly at low magnetic fields. Having in mind that static disorder plays a very similar role on the dynamics of magnetic and martensitic systems, we have undertaken as well a study of the correlation between the cycle-averaged fluctuations of the order parameter, $\overline{E^{2}}-\bar{E}^{2}$, and the cycle-averaged response function $d \bar{E} / d \tau$.

The study has been done for the five transformation trajectories shown on top of Fig. 13, in the limit of intensive cycling (SEV) for which the disorder is assumed to remain static. Each loading trajectory can be regarded as a route in the phase space of all possible martensite domain arrangements. Presumably, the trajectories differ from each other due to thermal fluctuations. ${ }^{24}$ Such fluctuations exist, since the system is not strictly at $T=0$. Following Urbach et al., the observed statistical reproducibility of the trajectories is a measure of the extent to which the disorder quenched in the system limits the divergence of the different routes in phase space.

In spite of the modest statistics, we have found the remarkable correlation between energy fluctuations and nonequilibrium response function shown in Fig. 13 (bottom). The correlation reflects the fact that $E-\tau$ trajectories tend to fluctuate more strongly, from cycle to cycle, at those stress fields where a large number of avalanches of relatively high amplitude occur and, conversely, to fluctuate less where avalanches are rare and have low amplitude. These observations point to a scenario where some defects are powerful enough to pin the transformation in an equivalent, reproducible way in each cycle, while the pinning power of weaker defects is subject to thermal fluctuations and differs appreciably from cycle to cycle.

The correlation observed is reminiscent of a fluctuation- 
dissipation theorem (out of equilibrium), and hence indicative of the fact that the behavior of the system is ergodic, at least in a restricted portion of phase space. This is again a question that would merit theoretical investigation.

\section{CONCLUSIONS}

We have presented an experimental study of the dynamics of the stress-induced formation of martensite in a $\mathrm{Cu}-\mathrm{Zn}-\mathrm{Al}$ single crystal. The geometry of the transition is particularly simple in this situation, since only one martensite variant is selected. Our results confirm that the transition is athermal, i.e., nearly insensitive to thermal fluctuations, and requires a monotonic variation of the applied load to be driven. In the transition, the crystal is taken from the original bcc phase into the close-packed martensitic structure through a sequence of long-lived metastable two-phase states. These states are separated by large energy barriers, due to the building up of long range elastic strain fields caused by the presence of crystalline defects and by lattice shape incompatibilities between the two phases. This kind of behavior has its macroscopic manifestation on the thermal power released or absorbed by the crystal (basically related to the latent heat of transition), recorded as a function of applied load: the recording displays an irregular sequence of sharp thermal events separated by relatively large quiet intervals, characteristic of the stick-slip behavior described.

The statistical distributions of amplitudes, durations and energies of the thermal peaks recorded in the first fifty transition cycles have been found to follow power laws over the range experimentally accessible (one and a half to two decades). This result indicates the absence of characteristic scales in the transition for these first transformation cycles. Upon cycling, however, the largest events tend to disappear. The transition tends to spread over a larger range of applied load than in the first cycles, and to form a larger number of martensite domains, finer than before. After five hundred transition cycles the extent of the power laws is reduced by at least one decade and the system becomes subcritical.

Considering that the interval of applied load between consecutive transformation events is a measure of the energy barrier between the coresponding metastable configurations, we have been able to measure the distribution of energy barrier sizes encountered by the system along the phase transition. Remarkably we find barrier sizes extending over more than four decades in energy, indicative of the complexity of the free energy landscape. Our results show that the amount of large energy barriers is nearly unchanged with cycling, while that of low and intermediate barriers has nearly doubled after five hundred cycles.

In the limit where the sample has undergone a large number of cycles and the transformation trajectories become statistically reproducible, we have found a remarkable correlation between cycle-to-cycle trajectory fluctuations and the cycle-averaged response function of the system, which is in complete analogy with the correlation found between cycleaveraged fluctuations of magnetization and the cycleaveraged susceptibility of a magnetic system. ${ }^{17}$

\section{ACKNOWLEDGMENTS}

The authors are indebted to Eduard Vives and Antoni Planes for many helpful comments, and to Per-Anker Lindgard for suggestions concerning the experimental setup. The technical help provided by Daniel Binagüi, from the Serveis Científico-Tècnics of the University of Barcelona, is gratefully acknowledged. Ll.C. is supported by the Dirección General de Investigación Científica y Técnica (DGICyT, Spain). The work has received financial support of the Comisión Interministerial de Ciencia y Tecnología (CICyT, Spain), Project No. MAT95-0504, the Dirección General de Investigación Científica y Técnica (DGICyT, Spain), Project No. PB93-0054-C02-01, and the Comissionat d'Universitats i Recerca (Generalitat de Catalunya), Project No. GRQ1012.
${ }^{1}$ J. P. Sethna, K. Dahmen, S. Kartha, J. A. Krumhansl, B. W. Roberts, and J. D. Shore, Phys. Rev. Lett. 70, 3347 (1993); K. Dahmen and J. P. Sethna, ibid. 71, 3222 (1993); J. P. Sethna, K. Dahmen, S. Kartha, J. A. Krumhansl, O. Perkovic, B. W. Roberts, and J. D. Shore, ibid. 72, 947 (1994); O. Perkovic, K. Dahmen, and J. P. Sethna, ibid. 75, 4528 (1995); K. Dahmen and J. P. Sethna, Phys. Rev. B 53, 14872 (1996).

${ }^{2}$ J. A. Barker, D. E. Schreiber, B. G. Huth, and D. H. Everett, Proc. R. Soc. London, Ser. A 386, 251 (1983); D. C. Jiles and D. L. Atherton, J. Appl. Phys. 55, 2115 (1984).

${ }^{3}$ J. Ortín, J. Appl. Phys. 71, 1454 (1992), and references therein.

${ }^{4}$ In adsorption of gases by porous solids: J. Katz, J. Phys. Chem. 53, 1166 (1949); in soil-water sorption and desorption processes: D. Hillel, Soil and Water: Physical Principles and Processes (Academic Press, New York, 1971), and references therein.

${ }^{5}$ M. P. Lilly, P. T. Finley, and R. B. Hallock, Phys. Rev. Lett. 71, 4186 (1993).

${ }^{6}$ Z. Z. Wang and N. P. Ong, Phys. Rev. B 34, 5967 (1986).

${ }^{7}$ E. W. Müller, H. Spiering, and P. Gütlich, J. Chem. Phys. 79, 1439 (1983).
${ }^{8}$ J. Goicoechea and J. Ortín, Phys. Rev. Lett. 72, 2203 (1994).

${ }^{9}$ W. Cao, J. A. Krumhansl, and R. J. Gooding, Phys. Rev. B 41, 11319 (1990).

${ }^{10}$ B. Tadic, Phys. Rev. Lett. 77, 3843 (1996).

${ }^{11}$ J. Goicoechea and J. Ortín, J. Phys. IV 5, C2-71 (1995).

${ }^{12}$ E. Vives and A. Planes, Phys. Rev. B 50, 3839 (1994).

${ }^{13}$ E. Vives, J. Goicoechea, J. Ortín, and A. Planes, Phys. Rev. E 52, R5 (1995).

${ }^{14}$ P. J. Cote and L. V. Meisel, Phys. Rev. Lett. 67, 1334 (1991); L. V. Meisel and P. J. Cote, Phys. Rev. B 46, 10822 (1992); K. P. O'Brien and M. B. Weissman, Phys. Rev. E 50, 3446 (1994); D. Spasojević, S. Bukvić, S. Milosević, and H. E. Stanley, ibid. 54, 2531 (1996).

${ }^{15}$ E. Vives, J. Ortín, Ll. Mañosa, I. Ràfols, R. Pérez-Magrané, and A. Planes, Phys. Rev. Lett. 72, 1694 (1994); E. Vives, I. Ràfols, Ll. Mañosa, J. Ortín, and A. Planes, Phys. Rev. B 52, 12644 (1995).

${ }^{16}$ S. Field, J. Witt, F. Nori, and X. Ling, Phys. Rev. Lett. 74, 1206 (1995).

${ }^{17}$ J. S. Urbach, R. C. Madison, and J. T. Markert, Phys. Rev. Lett. 75, 4694 (1995). 
${ }^{18}$ J. Cahn, Prog. Mater. Sci. 36, 149 (1992).

${ }^{19}$ Ll. Mañosa, A. Planes, D. Rouby, and J. L. Macqueron, Acta Metall. Mater. 38, 1635 (1990).

${ }^{20}$ J. Ortín and A. Planes, Acta Metall. 37, 1433 (1989).

${ }^{21}$ I. Ràfols and E. Vives, Phys. Rev. B 52, 12651 (1995).

${ }^{22}$ J. Ortín and J. Goicoechea (unpublished).

${ }^{23}$ The distribution of energy barriers in a fluctuationless RFIM turns out to be remarkably similar to the experimental distributions reported here [J. Goicoechea (private communication)].
${ }^{24}$ Although we have considered thermal fluctuations negligible, and have been talking about athermal dynamics, this is only an approximation. Note that the action of the external field between transformation events is to reduce monotonically the height of the energy barriers. Thus, before the lowest energy barrier is effectively suppressed, thermal fluctuations of very low amplitude can become operative to make the system reach a new metastable configuration, through a new transformation event. 